\title{
Sesamin Protects the Femoral Head From Osteonecrosis by Inhibiting ROS-Induced Osteoblast Apoptosis in Rat Model
}

\author{
Shuang Deng, Jian-Lin Zhou, Hong-Song Fang, Zhi-Gang Nie, Sen Chen and Hao Peng* \\ Department of Orthopedics, Renmin Hospital of Wuhan University, Wuhan, China
}

Glucocorticoids intake has become the most common pathogenic factor for osteonecrosis of the femoral head (ONFH). Annually, tens of millions of patients suffer from pain related to ONFH. Researchers have proposed several underlying mechanisms of ONFH, including osteocyte apoptosis, cell differentiation disorder, and angiogenesis hindrance. Sesamin, isolated from Sesamum indicum seeds, was reported could affect osteocyte inflammation and differentiation in osteoarthritis and osteoporosis. We investigated the underlying influence of sesamin on ONFH rat model. Fifteen male

OPEN ACCESS

Edited by: George Grant University of Aberdeen, United Kingdom

Reviewed by:

Prachya Kongtawelert, Chiang Mai University, Thailand

Hermizi Hapidin,

Universiti Sains Malaysia, Malaysia

Dewei Zhao,

Affiliated Zhongshan Hospital of Dalian University, China

*Correspondence: Hao Peng penghao58688@163.com

Specialty section:

This article was submitted to Clinical and Translational Physiology, a section of the journal

Frontiers in Physiology

Received: 22 September 2018 Accepted: 28 November 2018

Published: 11 December 2018

Citation:

Deng S, Zhou J-L, Fang H-S, Nie Z-G, Chen S and Peng H (2018) Sesamin Protects the Femoral Head

From Osteonecrosis by Inhibiting ROS-Induced Osteoblast Apoptosis in Rat Model. Front. Physiol. 9:1787.

doi: 10.3389/fphys.2018.01787 Sprague-Dawley rats were randomly divided into three groups. The ONFH model group only received the methylprednisolone (MPS) and lipopolysaccharide (LPS) injection to promote the development of ONFH. The sesamin treatment group was injected with sesamin, MPS, and LPS. The control group was untreated. Rats from above groups were sacrificed 4 weeks later. The effect of sesamin on ONFH rats was validated by H\&E staining. TUNEL staining showed that femoral head necrosis was attenuated by sesamin. Furthermore, the phosphorylation of Akt was increased and the downstream cellular apoptosis signal pathway was inhibited. Intracellular ROS level was decreased after sesamin treatment. In conclusion, our findings suggest that sesamin protects the femoral head from osteonecrosis by inhibiting ROS-induced osteoblast apoptosis.

Keywords: sesamin, ONFH, dexamethasone, ROS, apoptosis

\section{INTRODUCTION}

Approximately 20,000-30,000 patients in the United States are diagnosed as ONFH annually (Moya-Angeler et al., 2015). Most patients are young individuals between 20 and 40 years of age, who will suffer lifelong symptoms (Larson et al., 2018). ONFH is divided into two categories: traumatic and nontraumatic. Chronic glucocorticoid (GC) steroid administration, alcohol abuse, and virus infection are thought to be the leading causes of nontraumatic ONFH. Of these, the cumulative intake of glucocorticoids is the most common pathogenic factor: $9-40 \%$ of patients receiving long-term glucocorticoid therapy develop ONFH (Weinstein, 2012; Xu et al., 2018).

Many investigations into the ONFH pathogenic mechanisms have been reported. To date, vasoconstriction, apoptosis, oxidative stress, lipid metabolism disturbances, and disturbances of the coagulation-fibrinolysis system are thought to be the molecular mechanisms of ONFH (Zheng et al., 2014; Yang et al., 2016). The interaction of glucocorticoid receptor-related kinases and phosphatases

Abbreviations: Dex, dexamethasone, LPS, lipopolysaccharide; ONFH, osteonecrosis of the femoral head; ROS, reactive oxygen species. 
alters inflammation in GC-induced animal models, leading to side effects including ONFH (Beck et al., 2009). In murine osteoblastic MC3T3-E1 cells, proliferation was sustained at the G1 phase as a result of Dex stimulation, which activated cellular apoptosis ( $\mathrm{Li}$ et al., 2012). The elevation of ROS expression resulted in endoplasmic reticulum (ER) stress, and triggered the apoptosis of primary osteocytes in C57BL/6 mice (Sato et al., 2015). It is now widely accepted that ONFH is a multi-genesis disease, which requires the development of more animal models, more intensive study, and better therapeutic agents.

Originally identified in sesame (Sesamum indicum), sesamin is found in 30 different plants belonging to different genera, including Sesamum, Virola, Piper, Camellia, and Magnolia (Akl et al., 2013; Dou et al., 2018). Sesamin was reported to have various anti-cancer functions, including protecting cells from oxidative stress, reducing tumor cell proliferation, inhibiting inflammatory processes, and stimulating cellular apoptosis (Nakano et al., 2003; Hou et al., 2004; Fujikawa et al., 2005; Banjerdpongchai et al., 2010; Chung et al., 2010). Previous studies reported that sesamin suppressed tumor progress at the cellular level. In HepG2, a human hepatocellular carcinoma cell line, sesamin significantly inhibited proliferation by arresting cells at the G2/M phase, leading to the activation of STAT3 and the apoptosis pathway (Deng et al., 2013). In another study, researchers demonstrated that sesamin activated Bax, caspase-3, p53, and checkpoint kinase 2 (Siao et al., 2015). In contrast, cells were protected from inflammation in other studies. Sesamin attenuated inflammation in LPS-induced acute lung injury BALB/c mice by suppressing LPS-induced cytokine (TNF- $\alpha$, IL-6, and IL-1 $\beta$ ) production (Qiang et al., 2016). In TACinduced cardiac hypertrophy mouse models, sesamin suppressed hypertrophic and fibrotic responses via the Sirt3/ROS pathway (Fan et al., 2017).

The function of sesamin in osteoarthritis and osteoporosis has also been investigated. Sesamin inhibited IL- $1 \beta$-stimulated inflammatory responses in primary osteoarthritis chondrocytes by activating the Nrf2 signaling pathway (Kong et al., 2016). In M-CSF- and RANKL-induced human PBMCs, sesamin had a significant inhibitory effect on osteoclast differentiation (Wanachewin et al., 2017). However, no studies have reported the influence of sesamin in the ONFH model.

In this study, we investigated the function of sesamin in an $\mathrm{ONFH}$ rat model and primary osteoblasts. H\&E staining and TUNEL assay revealed that sesamin attenuated ONFH progression. Furthermore, experiments using primary osteoblasts indicated that Dex activated cellular apoptosis, which was reversed by sesamin, possibly by decreasing ROS expression. Our study is the first functional investigation of sesamin in $\mathrm{ONFH}$, and provides a wider perspective for disease treatment.

\section{MATERIALS AND METHODS}

\section{Animals}

This study was carried out in accordance with the recommendations of the Institutional Animal Care and Use Committee of Wuhan University, followed by the guidance for the Care and Use of Laboratory Animals (1996). The protocol was approved by the Institutional Animal Care and Use Committee of Wuhan University [No. WDRM (FU) 20180212]. A total of 15 male Sprague-Dawley rats aging at 8-10-week-old, weighing $250 \pm 20 \mathrm{~g}$, were got from the Hubei Provincial Center for Disease Control and Prevention, Wuhan, China. The rats were housed under specific pathogen-free (SPF) conditions. Besides, they were provided access to conventional chow and tap water ad libitum. All surgery was operated under anesthesia, and all attempts were made to minimize the suffering.

\section{Cell Culture}

Primary osteoblasts were obtained from neonatal rats. All procedures were performed under sterile conditions. Briefly, rats were soaked in $75 \%$ ethanol. After washing with sodium phosphate buffer (PBS, Hyclone), the skulls and periosteum were obtained. Blood vessels and connective tissues were removed. Then skulls were cut into pieces and transferred into a culture flask. Four milliliters of trypsin was added into the flask for 5 min. After digestion was completed, Ham's F-12 Nutrient Mixture (Hyclone) containing 10\% fetal bovine serum (FBS) (Gibco) was used to terminate the digestion. The skull pieces were incubated with $0.1 \%$ collagenase I solution for $30 \mathrm{~min}$; then centrifuged to collect the osteoblasts, which were cultured in Ham's F-12 Nutrient Mixture containing $10 \%$ FBS at $37^{\circ} \mathrm{C}$ with $5 \% \mathrm{CO}_{2}$. One hour later, the flask was turned over and the osteoblasts were continuously incubated. Once primary culture cells reached confluence over the entire bottom of the flask, they were ready for passage. Third generation cells were used in these experiments.

First, $1 \times 10^{8}$ primary osteoblasts were cultured in $25 \mathrm{~cm}$ dishes and different amounts of sesamin were added to the culture media for $24 \mathrm{~h}$. Then $1 \mu \mathrm{M}$ Dex (Sigma-Aldrich, St. Louis, MO, United States) was incubated with the cells for another $24 \mathrm{~h}$. Then cells were collected for further experiments.

\section{Materials}

Sesamin (catalog no. S171302, purity > 98\%) was purchased from Aladdin Industrial Corporation (Shanghai, China), and was suspended in $0.5 \%$ carboxymethylcellulose solution for animal experiments. Its structure was documented in PubChem (no. 24899834). LPS (Escherichia coli serotype 055: B5) and Dex were purchased from Sigma-Aldrich (St. Louis, MO, United States). Methylprednisolone (MPS) was purchased from Pfizer Pharmaceutical, China. Primary antibodies used in this study, rabbit anti-Bcl-2 polyclonal antibody (ab196495), rabbit anti-Bax monoclonal antibody (ab32503), rabbit antiAkt polyclonal antibody (ab8805), and rabbit anti-phosphoAkt polyclonal antibody (ab38449) were purchased from Abcam Biotechnology (Cambridge, MA, United States). Rabbit anti-caspase-3 polyclonal antibody and mouse anti-GAPDH monoclonal antibody (no. 60004), used as a loading control, were purchased from ProteinTech Biotechnology.

\section{Experimental Design}

In animal experiments, all rats were randomly divided into three groups. (1) Sesamin treatment group $(n=5)$ : rats were injected with sesamin, LPS, and MPS in the first week, and given $0.9 \%$ 
saline over the next 3 weeks. On days 1 and 2, rats were given an intraperitoneal injection of sesamin $(100 \mathrm{mg} / \mathrm{kg}) 2 \mathrm{~h}$ before the intravenous injection of LPS (1.8 mg/kg) (Kong et al., 2009, 2015; Zhang et al., 2013; Qiang et al., 2016). On days 3-7, the animals were given $25 \mathrm{mg} / \mathrm{kg}$ MPS intramuscularly $2 \mathrm{~h}$ after the intraperitoneal injection of sesamin to promote the development of femoral head necrosis as described by Okazaki et al. (2009, 2012). (2) ONFH model group $(n=5)$ : rats were given LPS and MPS in the same manner as the sesamin treatment group in the first week, and $0.9 \%$ saline was given in the same mode for another 3 weeks. (3) Control group $(n=5)$ : all rats were given $0.9 \%$ saline during the 4 weeks.

The animals were sacrificed at the end of the fourth week with an overdose injection of pentobarbital sodium. Subsequently, bilateral femoral heads from each rat were excised. The right femoral head was fixed in $4 \%$ paraformaldehyde, and the left femoral head was put in liquid nitrogen immediately and stored at $-80^{\circ} \mathrm{C}$ until analyzed.

\section{Hematoxylin and Eosin Staining}

Femoral head tissues were excised and fixed in neutral buffered solution for $48 \mathrm{~h}[4 \%$ formaldehyde in $0.1 \mathrm{M}$ sodium phosphate buffer (PBS), $\mathrm{pH}=7.4$ ], decalcified in $10 \%$ ethylenediaminetetraacetic acid (EDTA) for 4 weeks, dehydrated in graded alcohols, and finally embedded in paraffin. Paraffin (5 $\mu \mathrm{m})$ sections were mounted on glass slides and processed by hematoxylin and eosin ( $\mathrm{H} \& \mathrm{E})$ staining for general morphological evaluation. Three sections were obtained from each of five animals per group. A representative image is shown for each group.

\section{TUNEL Assay}

Apoptotic osteoblasts and osteocytes were detected using the terminal deoxynucleotidyl transferase-mediated dUTP nick endlabeling (TUNEL) assay, with an in Situ Cell Death Detection Kit (Roche Diagnostics, Mannheim, Germany), according to the manufacturer's instructions. Briefly, following routine deparaffinization and treatment with $\mathrm{H}_{2} \mathrm{O}_{2}(3 \%)$, the sections were digested with proteinase $\mathrm{K}(20 \mu \mathrm{g} / \mathrm{mL}, \mathrm{pH} 7.4,12 \mathrm{~min})$ at $25^{\circ} \mathrm{C}$ and incubated with the reaction mixture (1:40, $60 \mathrm{~min})$ at $37^{\circ} \mathrm{C}$. Incorporated fluorescein was detected with horseradish peroxidase following incubation for $30 \mathrm{~min}$ at $37^{\circ} \mathrm{C}$ and were subsequently dyed with $3,3^{\prime}$-diaminobenzidine (DAB). Brown nuclei were assessed as positive apoptotic cells.

\section{Western Blotting}

Osteoblasts were processed by M-PER mammalian protein extraction reagent (Pierce, Rockford, IL, United States) and protease inhibitor cocktail set III (Calbiochem, Darmstadt, Germany) plus $5 \mathrm{mmol} / \mathrm{L}$ EDTA. Overall, $20 \mu \mathrm{g}$ of protein was loaded and separated by $10 \%$ SDS-PAGE. Then, the separated proteins were transferred to a PVDF membrane and blocked with $5 \%$ non-fat milk in Tris-buffer saline containing $0.05 \%$ Tween-20 (TBST). The membranes were incubated with the corresponding primary antibodies for $1 \mathrm{~h}$ at room temperature or overnight at $4^{\circ} \mathrm{C}$. Then, the membranes were washed three times and incubated with a horseradish peroxidase-conjugated secondary antibody $(1: 10,000)$ in TBST for $1 \mathrm{~h}$ at room temperature. All specific bands were visualized by an ECL system kit (Pierce Biotechnology, Beijing, China) and the numerical value of the density was detected by ImageJ software (NIH, Bethesda, MD, United States). Additionally, densitometric data from each blot were standardized to the control conditions with the value set to one for each experiment and all tests were repeated three times.

\section{Quantitative Reverse Transcription PCR (qRT-PCR)}

Total RNAs in primary osteoblasts were isolated using Trizol reagent (Invitrogen, Carlsbad, CA, United States) according to the manufacturer's protocol. One microgram of extracted total RNA was used for reverse transcription. The M-MLV Reverse Transcriptase and RNas in Ribonuclease Inhibitor were purchased from Promega. Primers in this experiment were listed in Table 1. The targeted mRNA amount was measured by real-time PCR with GAPDH as an endogenous control. All experiments were repeated at least three times.

\section{Measurement of Intracellular ROS Levels}

Changes in the ROS level in primary osteoblasts were detected using $2^{\prime}, 7^{\prime}$-dichlorofluorescein-diacetate (DCFH-DA, SigmaAldrich, St. Louis, MO, United States) staining. Cells were

TABLE 1 | Real-time quantitative PCR primer sequences.

\begin{tabular}{ll}
\hline Gene & Sequences \\
\hline Bax & Forward: 5'-GGCGATGAACTGGACAACAA-3' \\
& Reverse: 5'-CAAAGTAGAAAAGGGCAACC-3' \\
Bcl-2 & Forward: 5'-GGTGAACTGGGGGAGGATTG-3' \\
& Reverse: 5' -GCATGCTGGGGCCATATAGT-3' \\
Caspase-3 & Forward: 5'-GGACCTGTGGACCTGAAAAA-3' \\
& Reverse: 5'-GCATGCCATATCATCGTCAG-3' \\
GAPDH & Forward: 5'-GAAGGTGAAGGTCGGAGTC-3' \\
& Reverse: 5'-GAAGATGGTGATGGGATTC-3'
\end{tabular}

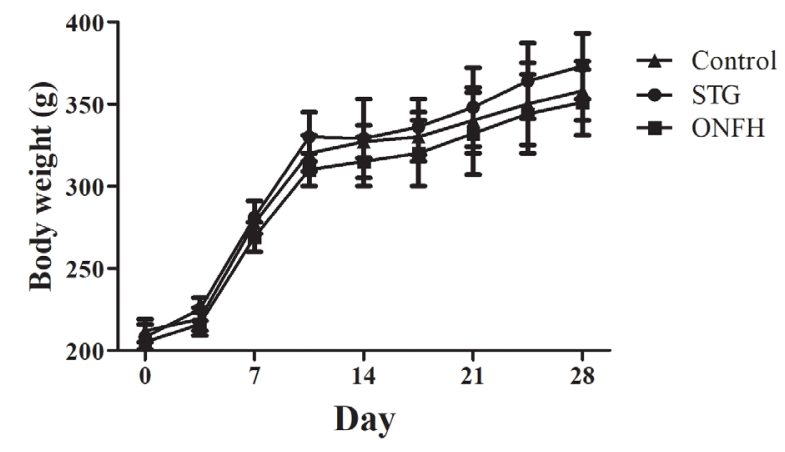

FIGURE 1 | Body weight over time of rats in the sesamin treatment group, ONFH model group, and control group. Five rats were used in each group. STG, sesamin treatment group; ONFH, ONFH model group; Control, control group. Data values are expressed as mean $\pm \mathrm{SD}$. 
seeded into a 24 -well plate with $3 \times 10^{3}$ cells per well and exposed to different treatments. Then, adherent and floating cells in each group were harvested, washed with PBS three times, and stained with $10 \mu \mathrm{M}$ DCFH-DA for $30 \mathrm{~min}$ at $37^{\circ} \mathrm{C}$ in the dark. The mean fluorescence intensity of each group was analyzed by flow cytometry (Bender MedSystems, Burlingame,
CA, United States), which represented the intracellular ROS level.

\section{Statistical Analysis}

In this study, all values were expressed as the mean \pm standard deviation (SD) of at least three independent experiments.
A

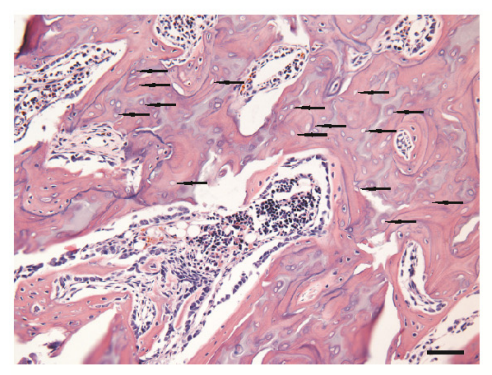

B

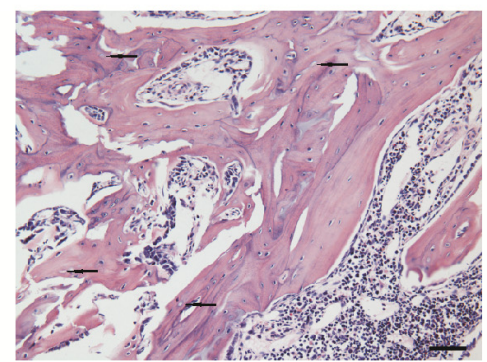

D

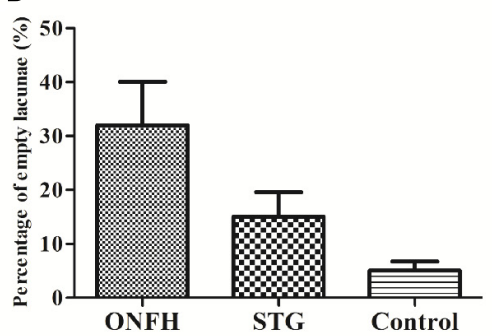

C

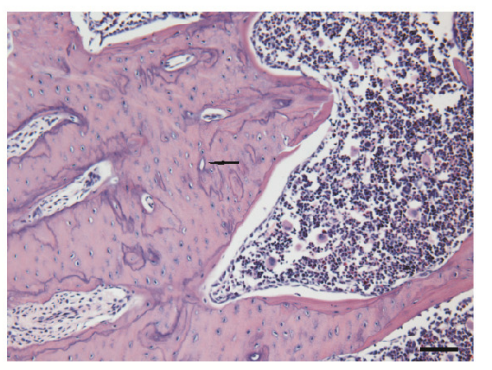

FIGURE 2 | Histological appearance of the femoral head in three groups. (A) ONFH model group. (B) Sesamin treatment group. (C) Control group. The arrows indicated the empty lacunae. The original magnification of HE staining is $200 \times$, and the scale bars were $100 \mu \mathrm{m}$. (D) The rate of empty lacunae in three groups. STG, sesamin treatment group; ONFH, ONFH model group; Control, control group. Data values are expressed as mean \pm SD.
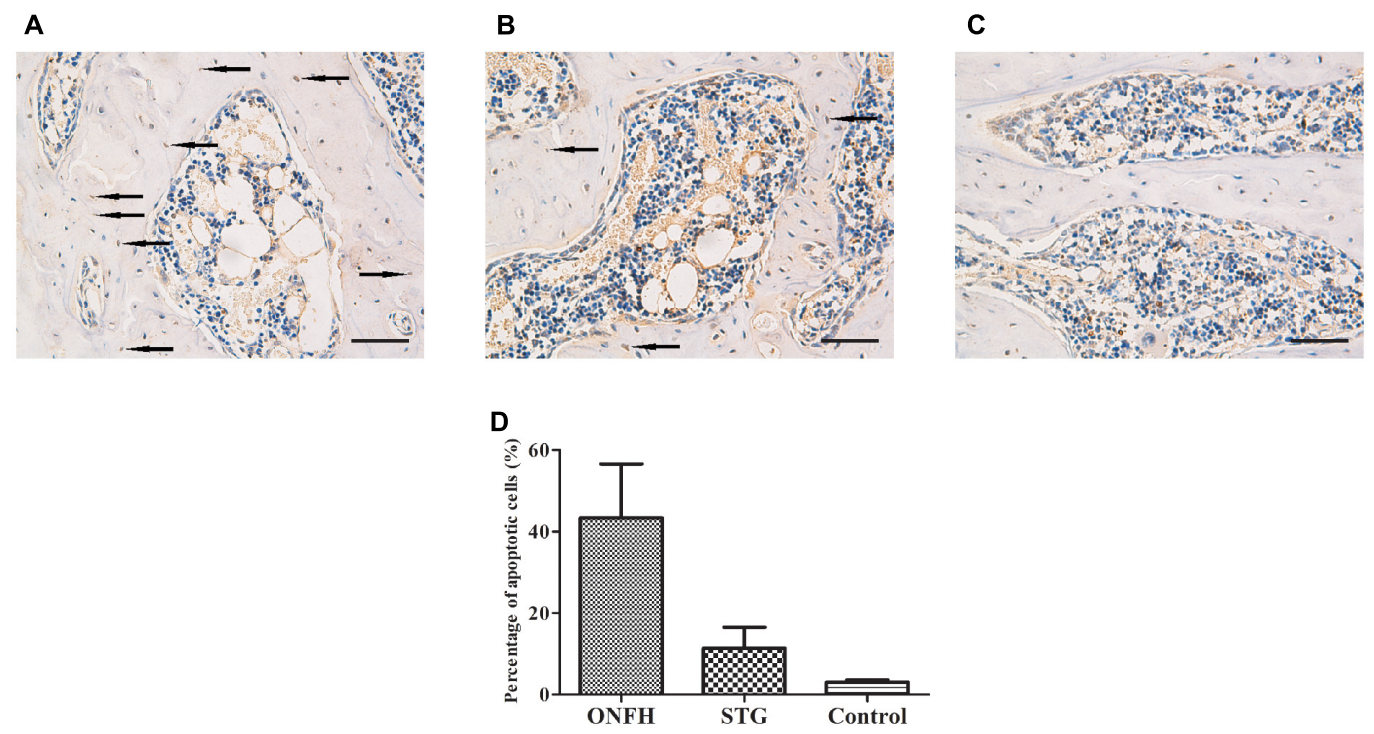

FIGURE 3 | Photomicrograph of TUNEL staining showing evidence of apoptotic cells in the necrotic zone. (A) ONFH model group. (B) Sesamin treatment group. (C) Control group. The arrows indicated the apoptotic cells. The original magnification of TUNEL staining is $200 x$, and the scale bars were $100 \mu \mathrm{m}$. (D) The percentage of apoptotic cells in three groups. STG, sesamin treatment group; ONFH, ONFH model group; Control, control group. Data values are expressed as mean \pm SD. 
Statistical analyses were performed using SPSS 17.0 software (SPSS Inc., Chicago, IL, United States). The Student's $t$-test was used for analyses and statistical significance was defined as $p<0.05$.

\section{RESULTS}

\section{Sesamin Attenuates Osteonecrosis in SD Rats}

To evaluate the influence of sesamin on ONFH rats, $100 \mathrm{mg} / \mathrm{kg}$ sesamin was intraperitoneally injected into normal rats, and then LPS and MPS were used to induce ONFH. The dose of sesamin used in this study was based on previous studies ( $\mathrm{Ma}$ et al., 2015; Qiang et al., 2016). Throughout the study, no significant side effects were induced by sesamin. The mean body weight was not significantly different between the groups (Figure 1), and no accidental death occurred during the whole study. When injected with LPS and MPS, the femoral head of rats developed severe osteonecrosis. However, sesamin appeared to relieve pain in ONFH rats as determined by behavioral observation (data not shown).

The necrosis of femoral head was determined by macroscopic morphology observation. In normal group, bilateral femoral head was spherical and not collapsed. The surface of cartilage was ruddy and glossy, and smooth with homogeneous thickness. While in ONFH model group, the surface of cartilage became dim in color and lack of gloss. It was rough with minimal fibrillation or a slight yellowish discoloration. Different degrees of wear occur in some area. Since the treatment of Dex lasted for only
4 weeks, no femoral head collapse or osteophyte formation was found in the necrotic femoral head.

As in our experiment, no necrotic feature was observed in any rat or femoral head in control group. In the ONFH model group, necrosis was found in all five rats. Four out of the five rats had bilateral necrosis femoral head and the other one had unilateral necrosis femoral head (the rate of necrosis was 9/10). While in sesamin treatment group, necrosis was found in four rats. Only two out of the five rats had bilateral necrosis femoral head and another two had unilateral necrosis femoral head (the rate of necrosis is 6/10).

Microscopic morphology tests were used to validate the effect of sesamin (Figure 2). H\&E staining was used to assess the general morphology of samples. In the ONFH model group (Figure 2A), the bone trabeculae showed pyknotic nuclei and empty lacunae, and fibrous tissues had accumulated in the medullary space, which are characteristic of severe osteonecrosis. Bone trabeculae in the sesamin treatment group (Figure 2B) rarely had pyknotic nuclei and empty lacunae suggesting osteonecrosis was inhibited. Besides, we calculated the rate of empty lacunae (Figure 2D). The results were counted from five randomly selected high-power fields in one section from each rat. These results correlated with the behavioral observations.

\section{Sesamin Reduces Apoptosis in the Osteonecrosis Zone}

To determine whether the administration of sesamin affected apoptosis in the trabecular bone of the femoral head, we conducted TUNEL assays. We found that positively stained cells were detected in the $\mathrm{ON}$ zones in the ONFH model group and

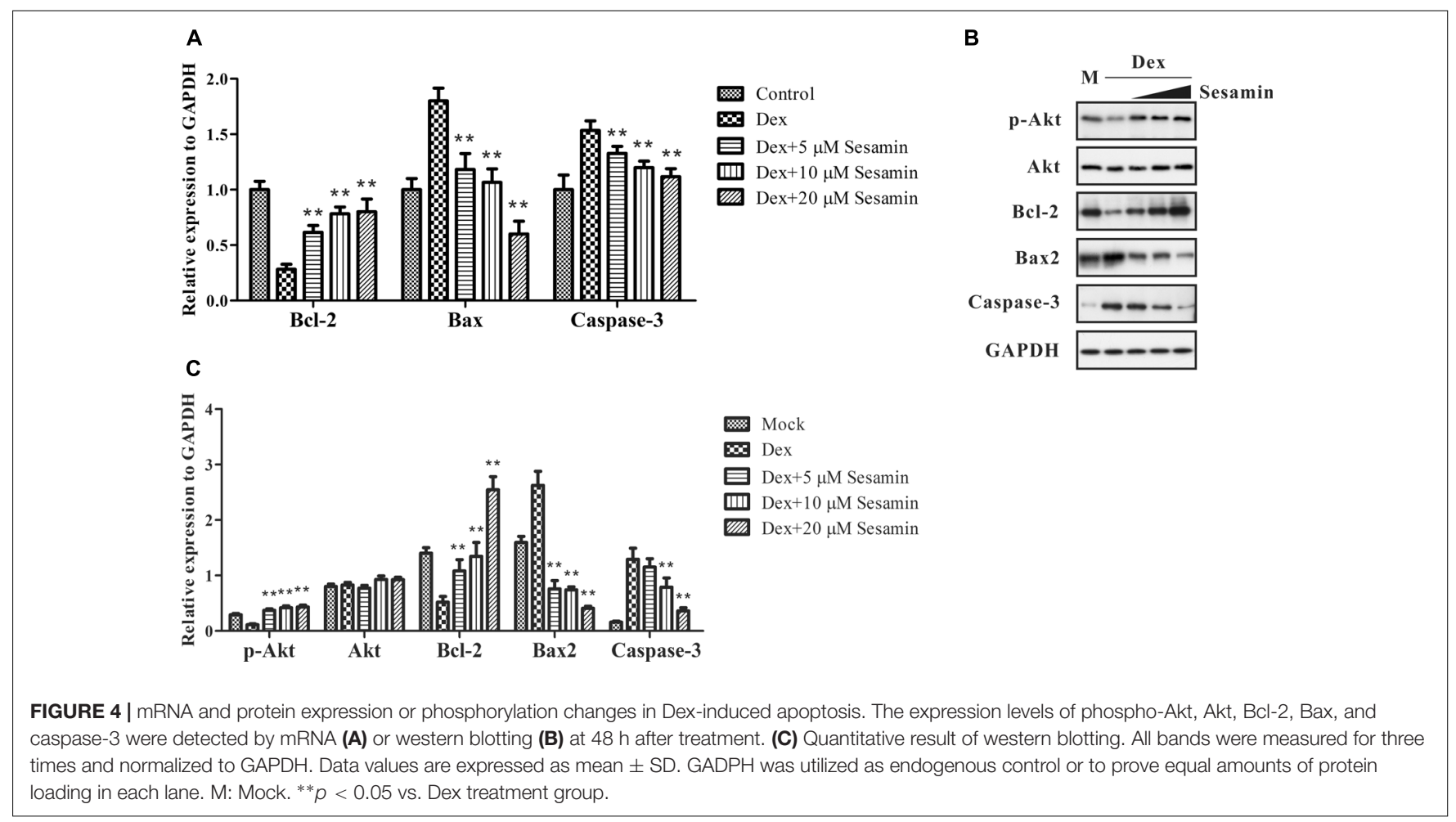


sesamin treated group (Figures 3A,B). However, the number of TUNEL-positive cells in high-power field was significantly reduced compared with that in $\mathrm{ONFH}$ model group (Figure 3C). Then we calculated the percentage of apoptotic cells in three groups (Figure 3D). The results were counted from five randomly selected high-power fields in one section from each rat. These data indicated that the administration of sesamin protected the trabecular bone cells from apoptosis.

\section{Osteoblast Apoptosis Is Inhibited by Sesamin}

Prior to the analysis of the effects of sesamin on primary osteoblasts, we investigated the potential for cytotoxicity. Previously, sesamin at various concentrations (1.75-28 $\mu \mathrm{M})$ and processing time $(24,48$, and $72 \mathrm{~h}$ ) were tested in a human fetal osteoblast cell line and showed that sesamin was not cytotoxic (Wanachewin et al., 2012). A similar result was obtained in another work using primary human articular chondrocytes (Phitak et al., 2012; Pothacharoen et al., 2014; Kong et al., 2016). In our study, cells were treated with 5,10 , and $20 \mu \mathrm{M}$ sesamin for $48 \mathrm{~h}$. No obvious cytotoxic effects were detected by MTT assay (data not shown).

In the Dex treatment group, $1 \times 10^{8}$ primary osteoblasts were incubated with $1 \mu \mathrm{M}$ Dex for $24 \mathrm{~h}$. For the sesamin treatment group, 5, 10, or $20 \mu \mathrm{M}$ sesamin was added into culture media at $24 \mathrm{~h}$ before Dex incubation, and cells were stimulated for another $24 \mathrm{~h}$. The control group did not receive any treatment. During Dex-induced apoptosis, the proportion of $\mathrm{Bax} / \mathrm{Bcl}-2$ was increased, and caspase- 3 was cleaved to induce apoptosis. Akt is upstream of $\mathrm{Bax} / \mathrm{Bcl}-2$, and the phosphorylation of Akt promotes the inhibition of apoptosis. Therefore, we detected the intracellular amounts of these proteins (Figure 4). The mRNA expression levels of Bax, Bcl-2, and caspase- 3 were detected by qRT-PCR (Figure 4A). The results showed that Bcl-2 was upregulated when the concentration of sesamin was increased, while Bax and caspase- 3 were downregulated in this process. Western blotting revealed that the phosphorylation of Akt was significantly upregulated in the sesamin treatment group compared with the Dex treatment group indicating that cellular apoptosis was inhibited (Figure 4B). Quantitative results of western blotting were measured in Figure 4C. This was confirmed by direct evidence that Bax and caspase- 3 were decreased while $\mathrm{Bcl}-2$ was increased. All the regulatory effects were dose dependent. Therefore, sesamin inhibits Dex-induced osteoblast apoptosis.

\section{Sesamin Reduces ROS Levels in Osteoblast}

A previous work study suggested ROS inhibits the PI3K/Akt pathway and induces osteoblast apoptosis after Dex treatment (Lu et al., 2008; Li et al., 2015; Sato et al., 2015). Therefore, we detected the intracellular ROS level using DCFH-DA staining. $1 \times 10^{8}$ primary osteoblasts were incubated with different doses $(5,10$, and $20 \mu \mathrm{M})$ of sesamin for $24 \mathrm{~h}$. After treatment with $1 \mu \mathrm{M}$ Dex for another $24 \mathrm{~h}$, osteoblasts were harvested. As shown in Figure 5, Dex increased ROS expression, but this effect was

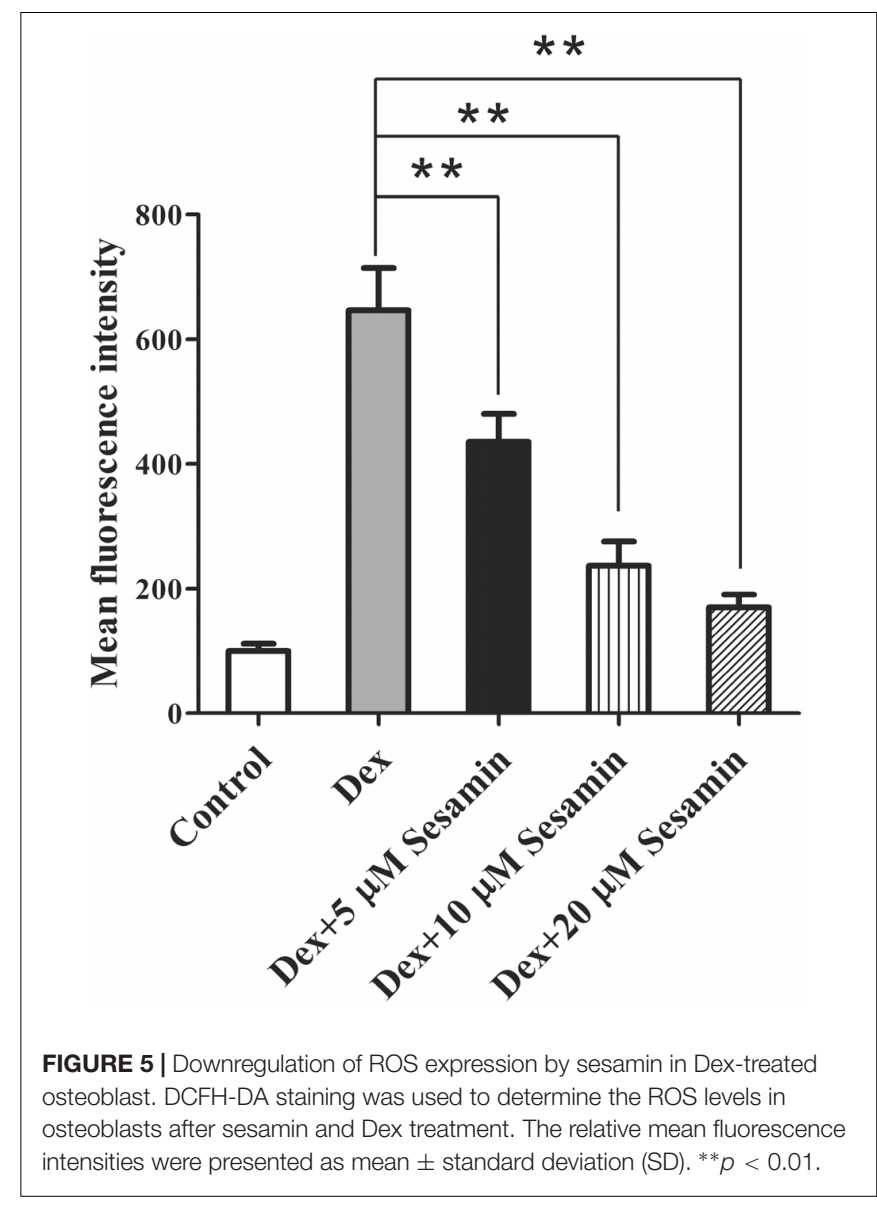

largely counteracted by sesamin, indicating that Dex-induced ROS expression was downregulated by sesamin.

\section{DISCUSSION}

Osteonecrosis of the femoral head has become a common disease with a high disability rate. Recently, there has been significant progression in diagnosis and therapeutics, yet its pathogenesis is still poorly understood. Among various hypotheses, apoptosis is one of the most studied mechanisms. Apoptotic signals transduced through the PI3K/Akt-Bax/Bcl-2/caspase 3 pathway has been reported in some orthopedic diseases ( $\mathrm{Li}$ et al., 2012, 2015; Sato et al., 2015). In our study, the protective function of sesamin on glucocorticoid-treated femoral head and osteoblast was confirmed. H\&E staining and TUNEL assay were used to demonstrate the influence of sesamin on glucocorticoidinduced ONFH. Akt-mediated apoptosis was inhibited and ROS expression was downregulated by sesamin. Taken together, we demonstrated the anti-apoptosis function of sesamin in femoral heads.

The accumulative administration of glucocorticoid accounts for dominant nontraumatic ONFH cases, but the pathogenesis of ONFH is still unknown. Noble et al. (1997) reported that osteocytes die by apoptosis. It is now widely accepted that the differentiation balance between osteoblasts and osteoclasts 
determines the fate of the femoral head (Weinstein, 2011, 2012; Jilka et al., 2013). Therefore, numerous studies have concentrated on osteoblast apoptosis. Phosphorylation activates Akt in osteoblasts to enhance the anti-apoptotic function of Bcl-2 (Ma et al., 2014). In our study, Akt was more active in the sesamin treatment group compared with the Dex treatment group. This suggests that sesamin affects cell apoptosis via the PI3K/Akt pathway.

A relationship between sesamin and apoptosis was demonstrated in breast cancer (Lee et al., 2011), hepatocellular carcinoma (Deng et al., 2013), cervical carcinoma (Dou et al., 2018), and leukemia (Banjerdpongchai et al., 2010). In contrast to our study, sesamin promoted cancer cell apoptosis to inhibit tumor genesis. This difference might be explained by the different cell types used in each study, because cancer cells show excessive proliferation. Sesamin contributes to the balance of cell differentiation and apoptosis. In cancer cells, sesamin decreases cell differentiation and proliferation, while in osteoblasts, apoptosis is inhibited to maintain the balance. This is supported by other reports in orthopedic diseases. In osteoporosis, sesamin promoted osteoblast differentiation in a human fetal osteoblast cell line via the MAPK signaling pathway (Wanachewin et al., 2012, 2017). Therefore, sesamin may positively regulate the MAPK signaling pathway in osteonecrosis; however, this requires further investigation.

The expression of ROS was elevated by Dex treatment. Excessive ROS may lead to cell apoptosis via the mitochondriamediated caspase apoptosis pathway (Ravindran et al., 2011). In addition, ROS accumulation causes oxidative stress and activates the JNK pathway in osteoblasts, which might inactivate the Akt pathway and promote osteoblast apoptosis (Cakir et al., 2011). In our study, ROS was significantly downregulated in the sesamin treatment group compared with the Dex treatment group. This indicated that the inhibitory effect of sesamin on osteonecrosis was related to mitochondria. In a study by Ratana, the mitochondrial membrane potential was reduced dosedependently in sesamin-treated U937 cells (Banjerdpongchai et al., 2010). However, whether sesamin acted as an anti-oxidant or pro-oxidant depended on the cell type and dose of sesamin. In our study, primary osteoblasts were used and the concentration of sesamin was 5-20 $\mu \mathrm{M}$. This is a frequently used concentration in human fetal osteoblast cell lines and primary human articular chondrocytes (Phitak et al., 2012; Wanachewin et al., 2012; Pothacharoen et al., 2014; Kong et al., 2016). The results of our cellular experiment correlated with the ONFH rat model.

\section{REFERENCES}

Akl, M. R., Ayoub, N. M., Abuasal, B. S., Kaddoumi, A., and Sylvester, P. W. (2013). Sesamin synergistically potentiates the anticancer effects of gamma-tocotrienol in mammary cancer cell lines. Fitoterapia 84, 347-359. doi: 10.1016/j.fitote. 2012.12.013

Banjerdpongchai, R., Yingyurn, S., and Kongtawelert, P. (2010). Sesamin Induces human leukemic cell apoptosis via mitochondrial and endoplasmic reticulum stress pathways. World J. Oncol. 1, 78-86. doi: 10.4021/wjon2010.03. $195 \mathrm{w}$

Beck, I. M., Vanden Berghe, W., Vermeulen, L., Yamamoto, K. R., Haegeman, G., and De Bosscher, K. (2009). Crosstalk in inflammation: the interplay of
Taken together, we suggest that sesamin is an antioxidant in osteonecrosis. Nevertheless, other cell lines and sesamin doses should be used to gain a better understanding of osteonecrosis.

Sesamin also regulates cell differentiation. It was reported that sesamin stimulated osteoblast differentiation (Wanachewin et al., 2012) and inhibited osteoclastogenesis (Wanachewin et al., 2017) via the p38/MAPK signaling pathway. Interestingly, the influence of sesamin on osteoblasts and osteoclasts is mediated via the phosphorylation regulation of p38 and ERK. Moreover, the concentration involved in both experiments was identical. Therefore, sesamin might be used in clinical therapeutics. Indeed, MAPK signaling regulates the fate of cells in many diseases. Li et al. (2016) demonstrated that sesamin inhibited the activation of p38/MAPK signaling in a human mast cell line to decrease mast cell-mediated allergic responses. In ischemic diseases, sesamin stimulated angiogenesis in vitro and in vivo through the activation of p38/MAPK pathways (Chung et al., 2010). Our study mainly studied the PI3K/Akt signaling pathway, which is involved in cellular apoptosis. Further studies should investigate the interactions between the PI3K/Akt and p38/MAPK pathways in osteonecrosis.

Glucocorticoid-induced ONFH has become a common public health problem. The effect of sesamin was reported in osteoarthritis and osteoporosis, but there were still no research in ONFH. In summary, our study reported a novel regulatory function of sesamin in osteonecrosis. We demonstrated that sesamin inhibited apoptosis in an ONFH rat model and primary osteoblasts, which may be related to ROS production. The findings suggest sesamin might be a therapeutic target for ONFH.

\section{AUTHOR CONTRIBUTIONS}

SD contributed conception and design of the study, finished most of the experiments, wrote the first draft of the manuscript, and completed the submission procedure. J-LZ, H-SF, and Z-GN helped with the animal experiments. SC performed the statistical analysis. All authors contributed to manuscript revision, read, and approved the submitted version.

\section{FUNDING}

This study was supported by the National Natural Science Foundation of China (No. 81672154).

glucocorticoid receptor-based mechanisms and kinases and phosphatases. Endocr. Rev. 30, 830-882. doi: 10.1210/er.2009-0013

Cakir, E., Yilmaz, A., Demirag, F., Oguztuzun, S., Sahin, S., Yazici, U. E., et al. (2011). Prognostic significance of micropapillary pattern in lung adenocarcinoma and expression of apoptosis-related markers: caspase-3, bcl-2, and p53. APMIS 119, 574-580. doi: 10.1111/j.1600-0463.2011.02778.x

Chung, B. H., Lee, J. J., Kim, J. D., Jeoung, D., Lee, H., Choe, J., et al. (2010). Angiogenic activity of sesamin through the activation of multiple signal pathways. Biochem. Biophys. Res. Commun. 391, 254-260. doi: 10.1016/j.bbrc. 2009.11.045

Deng, P., Wang, C., Chen, L., Wang, C., Du, Y., Yan, X., et al. (2013). Sesamin induces cell cycle arrest and apoptosis through the inhibition of signal 
transducer and activator of transcription 3 signalling in human hepatocellular carcinoma cell line HepG2. Biol. Pharm. Bull. 36, 1540-1548. doi: 10.1248/bpb. b13-00235

Dou, H., Yang, S., Hu, Y., Xu, D., Liu, L., and Li, X. (2018). Sesamin induces ER stress-mediated apoptosis and activates autophagy in cervical cancer cells. Life Sci. 200, 87-93. doi: 10.1016/j.lfs.2018.03.003

Fan, D., Yang, Z., Liu, F. Y., Jin, Y. G., Zhang, N., Ni, J., et al. (2017). Sesamin protects against cardiac remodeling via sirt3/ros pathway. Cell Physiol. Biochem. 44, 2212-2227. doi: 10.1159/000486026

Fujikawa, T., Kanada, N., Shimada, A., Ogata, M., Suzuki, I., Hayashi, I., et al. (2005). Effect of sesamin in Acanthopanax senticosus HARMS on behavioral dysfunction in rotenone-induced parkinsonian rats. Biol. Pharm. Bull. 28, 169-172. doi: 10.1248/bpb.28.169

Hou, R. C., Wu, C. C., Yang, C. H., and Jeng, K. C. (2004). Protective effects of sesamin and sesamolin on murine BV-2 microglia cell line under hypoxia. Neurosci. Lett. 367, 10-13. doi: 10.1016/j.neulet.2004.05.073

Jilka, R. L., Noble, B., and Weinstein, R. S. (2013). Osteocyte apoptosis. Bone 54, 264-271. doi: 10.1016/j.bone.2012.11.038

Kong, P., Chen, G., Jiang, A., Wang, Y., Song, C., Zhuang, J., et al. (2016). Sesamin inhibits IL-1beta-stimulated inflammatory response in human osteoarthritis chondrocytes by activating Nrf2 signaling pathway. Oncotarget 7, 83720-83726. doi: 10.18632/oncotarget.13360

Kong, X., Wang, G. D., Ma, M. Z., Deng, R. Y., Guo, L. Q., Zhang, J. X., et al. (2015). Sesamin ameliorates advanced glycation end products-induced pancreatic betacell dysfunction and apoptosis. Nutrients 7, 4689-4704. doi: 10.3390/nu7064689

Kong, X., Yang, J. R., Guo, L. Q., Xiong, Y., Wu, X. Q., Huang, K., et al. (2009). Sesamin improves endothelial dysfunction in renovascular hypertensive rats fed with a high-fat, high-sucrose diet. Eur. J. Pharmacol. 620, 84-89. doi: 10.1016/j. ejphar.2009.08.023

Larson, E., Jones, L. C., Goodman, S. B., Koo, K. H., and Cui, Q. (2018). Early-stage osteonecrosis of the femoral head: where are we and where are we going in year 2018? Int. Orthop. 42, 1723-1728. doi: 10.1007/s00264-018-3917-8

Lee, C. C., Liu, K. J., Wu, Y. C., Lin, S. J., Chang, C. C., and Huang, T. S. (2011). Sesamin inhibits macrophage-induced vascular endothelial growth factor and matrix metalloproteinase- 9 expression and proangiogenic activity in breast cancer cells. Inflammation 34, 209-221. doi: 10.1007/s10753-010-9226-Z

Li, H., Qian, W., Weng, X., Wu, Z., Li, H., Zhuang, Q., et al. (2012). Glucocorticoid receptor and sequential P53 activation by dexamethasone mediates apoptosis and cell cycle arrest of osteoblastic MC3T3-E1 cells. PLoS One 7:e37030. doi: 10.1371/journal.pone.0037030

Li, J., He, C., Tong, W., Zou, Y., Li, D., Zhang, C., et al. (2015). Tanshinone IIA blocks dexamethasone-induced apoptosis in osteoblasts through inhibiting Nox4-derived ROS production. Int. J. Clin. Exp. Pathol. 8, 13695-13706.

Li, L. C., Piao, H. M., Zheng, M. Y., Lin, Z. H., Li, G., and Yan, G. H. (2016). Sesamin attenuates mast cell-mediated allergic responses by suppressing the activation of p38 and nuclear factor-kappaB. Mol. Med. Rep. 13, 536-542. doi: 10.3892/mmr.2015.4546

Lu, P. Z., Lai, C. Y., and Chan, W. H. (2008). Caffeine induces cell death via activation of apoptotic signal and inactivation of survival signal in human osteoblasts. Int. J. Mol. Sci. 9, 698-718. doi: 10.3390/ijms9050698

Ma, L., Gong, X., Kuang, G., Jiang, R., Chen, R., and Wan, J. (2015). Sesamin ameliorates lipopolysaccharide/d-galactosamine-induced fulminant hepatic failure by suppression of Toll-like receptor 4 signaling in mice. Biochem. Biophys. Res. Commun. 461, 230-236. doi: 10.1016/j.bbrc.2015.03.154

Ma, P., Gu, B., Xiong, W., Tan, B., Geng, W., Li, J., et al. (2014). Glimepiride promotes osteogenic differentiation in rat osteoblasts via the PI3K/Akt/eNOS pathway in a high glucose microenvironment. PLoS One 9:e112243. doi: 10. 1371/journal.pone. 0112243

Moya-Angeler, J., Gianakos, A. L., Villa, J. C., Ni, A., and Lane, J. M. (2015). Current concepts on osteonecrosis of the femoral head. World J. Orthop. 6, 590-601. doi: 10.5312/wjo.v6.i8.590

Nakano, D., Itoh, C., Ishii, F., Kawanishi, H., Takaoka, M., Kiso, Y., et al. (2003). Effects of sesamin on aortic oxidative stress and endothelial dysfunction in deoxycorticosterone acetate-salt hypertensive rats. Biol. Pharm. Bull. 26, 17011705. doi: $10.1248 / \mathrm{bpb} .26 .1701$

Noble, B. S., Stevens, H., Loveridge, N., and Reeve, J. (1997). Identification of apoptotic changes in osteocytes in normal and pathological human bone. Bone 20, 273-282. doi: 10.1016/S8756-3282(96)00365-1
Okazaki, S., Nagoya, S., Tateda, K., Katada, R., Mizuo, K., Watanabe, S., et al. (2012). Weight bearing does not contribute to the development of osteonecrosis of the femoral head. Int. J. Exp. Pathol. 93, 458-462. doi: 10.1111/j.1365-2613.2012. 00836.x

Okazaki, S., Nishitani, Y., Nagoya, S., Kaya, M., Yamashita, T., and Matsumoto, H. (2009). Femoral head osteonecrosis can be caused by disruption of the systemic immune response via the toll-like receptor 4 signalling pathway. Rheumatology 48, 227-232. doi: 10.1093/rheumatology/ken462

Phitak, T., Pothacharoen, P., Settakorn, J., Poompimol, W., Caterson, B., and Kongtawelert, P. (2012). Chondroprotective and anti-inflammatory effects of sesamin. Phytochemistry 80, 77-88. doi: 10.1016/j.phytochem.2012. 05.016

Pothacharoen, P., Najarus, S., Settakorn, J., Mizumoto, S., Sugahara, K., and Kongtawelert, P. (2014). Effects of sesamin on the biosynthesis of chondroitin sulfate proteoglycans in human articular chondrocytes in primary culture. Glycoconj. J. 31, 221-230. doi: 10.1007/s10719-013-9514-6

Qiang, L., Yuan, J., Shouyin, J., Yulin, L., Libing, J., and Jian-An, W. (2016). Sesamin attenuates lipopolysaccharide-induced acute lung injury by inhibition of TLR4 signaling pathways. Inflammation 39, 467-472. doi: 10.1007/s10753015-0270-6

Ravindran, J., Gupta, N., Agrawal, M., Bala Bhaskar, A. S., and Lakshmana Rao, P. V. (2011). Modulation of ROS/MAPK signaling pathways by okadaic acid leads to cell death via, mitochondrial mediated caspase-dependent mechanism. Apoptosis 16, 145-161. doi: 10.1007/s10495-010-0554-0

Sato, A. Y., Tu, X., McAndrews, K. A., Plotkin, L. I., and Bellido, T. (2015). Prevention of glucocorticoid induced-apoptosis of osteoblasts and osteocytes by protecting against endoplasmic reticulum (ER) stress in vitro and in vivo in female mice. Bone 73, 60-68. doi: 10.1016/j.bone.2014.12.012

Siao, A. C., Hou, C. W., Kao, Y. H., and Jeng, K. C. (2015). Effect of sesamin on apoptosis and cell cycle arrest in human breast cancer mcf-7 cells. Asian Pac. J. Cancer Prev. 16, 3779-3783. doi: 10.7314/APJCP.2015.16.9.3779

Wanachewin, O., Boonmaleerat, K., Pothacharoen, P., Reutrakul, V., and Kongtawelert, P. (2012). Sesamin stimulates osteoblast differentiation through p38 and ERK1/2 MAPK signaling pathways. BMC Complement. Altern. Med. 12:71. doi: 10.1186/1472-6882-12-71

Wanachewin, O., Pothacharoen, P., Kongtawelert, P., and Phitak, T. (2017). Inhibitory effects of sesamin on human osteoclastogenesis. Arch. Pharm. Res. 40, 1186-1196. doi: 10.1007/s12272-017-0926-x

Weinstein, R. S. (2011). Clinical practice. glucocorticoid-induced bone disease. N. Engl. J. Med. 365, 62-70. doi: 10.1056/NEJMcp1012926

Weinstein, R. S. (2012). Glucocorticoid-induced osteonecrosis. Endocrine 41, 183190. doi: 10.1007/s12020-011-9580-0

Xu, J., Gong, H., Lu, S., Deasey, M. J., and Cui, Q. (2018). Animal models of steroidinduced osteonecrosis of the femoral head-a comprehensive research review up to 2018. Int. Orthop. 42, 1729-1737. doi: 10.1007/s00264-018-3956-1

Yang, Z., Liu, H., Li, D., Xie, X., Qin, T., Ma, J., et al. (2016). The efficacy of statins in preventing glucocorticoid-related osteonecrosis in animal models: a meta-analysis. Bone Joint Res. 5, 393-402. doi: 10.1302/2046-3758.59.20 00500

Zhang, J. X., Yang, J. R., Chen, G. X., Tang, L. J., Li, W. X., Yang, H., et al. (2013). Sesamin ameliorates arterial dysfunction in spontaneously hypertensive rats via downregulation of NADPH oxidase subunits and upregulation of eNOS expression. Acta. Pharmacol. Sin. 34, 912-920. doi: 10.1038/aps.2013.1

Zheng, H., Yang, E., Peng, H., Li, J., Chen, S., Zhou, J., et al. (2014). Gastrodin prevents steroid-induced osteonecrosis of the femoral head in rats by antiapoptosis. Chin. Med. J. 127, 3926-3931.

Conflict of Interest Statement: The authors declare that the research was conducted in the absence of any commercial or financial relationships that could be construed as a potential conflict of interest.

Copyright $\odot 2018$ Deng, Zhou, Fang, Nie, Chen and Peng. This is an open-access article distributed under the terms of the Creative Commons Attribution License (CC BY). The use, distribution or reproduction in other forums is permitted, provided the original author(s) and the copyright owner(s) are credited and that the original publication in this journal is cited, in accordance with accepted academic practice. No use, distribution or reproduction is permitted which does not comply with these terms. 